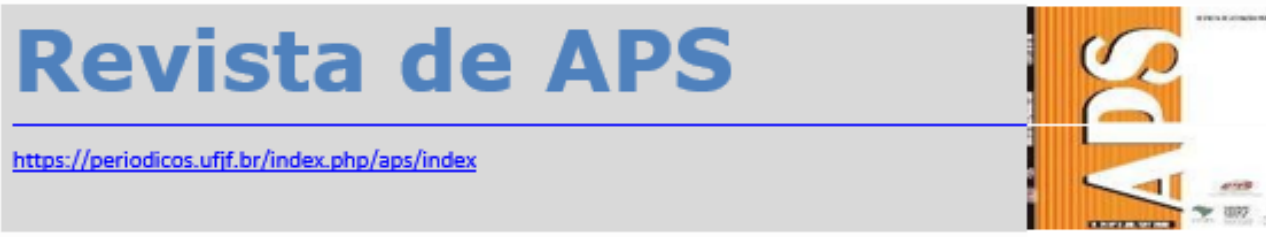

\title{
Implantação de um serviço sobre orientação de insulina na transição do cuidado: contribuições para o autocuidado
}

\author{
Setting up a service for insulin orientation during the transition in care: \\ contributions to self-care
}

\author{
Pollyanna Evelyn Ferreira Freitas ${ }^{1}$, Josiane Moreira Costa ${ }^{2}$, Ciomara Maria Pérez \\ Nunes ${ }^{3}$
}

\begin{abstract}
RESUMO
O diabetes mellitus é uma doença crônica responsável por inúmeras complicações que comprometem a qualidade de vida dos pacientes, sendo necessário para prevenção a adoção de medidas educativas que enfatize a importância do autocuidado e da adesão ao tratamento. Objetivo deste artigo é descrever as ações realizadas e os resultados alcançados com a implementação do Serviço de Orientação Sobre o Uso de Insulina (SOSUI). Trata-se de um estudo observacional, realizado no período de agosto de 2014 a fevereiro de 2015. Foram selecionados pacientes diabéticos insulinizados, sendo eles idosos e adultos frágeis submetidos à amputação devido a comprometimento de pé diabético. O SOSUI foi constituído em duas fases, sendo uma no período da internação hospitalar (pré-alta), e a segunda no período pós-alta hospitalar. No período pré-hospitalar, farmacêuticos vinculados a um programa de residência multiprofissional realizaram uma abordagem entre os pacientes, seguida de treinamentos práticos individualizados sobre a administração da insulina. Passado um período mínimo de quatro meses após a alta hospitalar, foi realizada interação mediada por telefone com o intuito de identificar mudanças nos relatos dos pacientes em relação ao uso da insulina, assim como melhora nos valores glicêmicos. Os dados foram registrados no programa Microsoft Excel, seguido de análise univariada. Foram 17 pacientes elegíveis para o estudo, com idade média de 63 anos, sendo $76,47 \%$ do sexo masculino e $23,53 \%$ do sexo feminino. 0 tempo médio de internação foi de 21 dias. Em relação ao perfil glicêmico dos participantes, observa-se que houve melhora nos níveis de glicemia de jejum e hemoglobina glicada dos pacientes orientados pelo serviço, e que estavam sob os cuidados da Atenção Primária. Além de melhoras nos
\end{abstract}

\footnotetext{
${ }^{1}$ Farmacêutica residente no Hospital Risoleta Tolentino Neves (MG).

2 Preceptora de estágio na Universidade Federal de Minas Gerais (UFMG).

${ }^{3}$ Professora na Universidade Federal de Minas Gerais (UFMG).
} 
relatos sobre técnicas de homogeneização e administração da insulina, foi evidenciada entre os pacientes uma hemoglobina glicada média de 9,97\%, sendo esse valor médio reduzido para 7,0\% após a alta hospitalar. Isso indica melhora do controle glicêmico pelos pacientes, o que pode ser influenciado pelas experiências de adoecimento, internação e amputação decorrente de complicações do diabetes, associadas ao fato de os pacientes terem também recebido orientações educacionais sobre o uso da insulina, favorecendo melhor entendimento sobre o uso desse medicamento. A implementação do SOSUI serviu como importante ferramenta no processo de educação em saúde sobre o diabetes para os pacientes acompanhados pelo Serviço de Farmácia Clínica, proporcionando melhora no gerenciamento da farmacoterapia com insulina; melhor conhecimento sobre o diabetes e suas complicações; e para a importância da adesão ao tratamento.

PALAVRAS-CHAVE: Diabetes. Insulina. Adesão ao tratamento.

\section{ABSTRACT}

Diabetes mellitus is a chronic disease that causes numerous complications that impair the quality of life of patients, making the adoption of educational measures that emphasize the importance of self-care and adherence to treatment necessary for prevention. Objective: To describe the actions and results achieved with the implementation of the Insulin Usage Guidance Service(SOSUI). Methodology: This is a observational study conducted from August / 2014 to February / 2015. Diabetic patients treated with insulin were selected, all of them elderly and vulnerable adults subjected to amputation due to pre-diabetic impairment. SOSUI constituted of two phases, one of them being during the hospitalization period (pre-discharge), and the second one being after hospital discharge. During the hospitalization period, pharmacists part of a multidisciplinary residency program approached patients, followed by individual practical training on insulin handling. After a period of at least 4 months of hospital discharge, a post-discharge contact was performed, mediated by phone to identify changes in the accounts of patients regarding insulin handling as well as improvement on glycemic levels. The data was recorded on Microsoft Excel, followed by univariate analysis. Results: 17 of the participants qualified for the study, with average age of $67,76.47 \%$ were male and $23.53 \%$ female. The average length of hospitalization was 21 days. Regarding the glycemic profile of the participants, it was observed that there was an improvement in fasting glucose levels and glycated hemoglobin of patients guided by the service, and under the care of Primary Care. Conclusion: The implementation of SOIRI served as an important tool in the health education process about diabetes for patients guided by the Clinical Pharmacy Service providing an improvement of the management of pharmacotherapy with insulin, a better knowledge about diabetes, its complications and the importance of adherence to treatment.

KEYWORDS: Diabetes. Insulin. Treatment adherence.

\section{INTRODUÇÃO}

A Organização Mundial da Saúde (OMS) define o diabetes mellitus (DM) como um distúrbio metabólico de etiologias diversas que se caracteriza por episódios de 
hiperglicemia e disfunção no metabolismo de carboidratos, proteínas e gorduras, provenientes da secreção e/ou ação inadequada do hormônio insulina. ${ }^{1}$

O diabetes é considerado uma doença crônica não transmissível, sendo uma epidemia mundial em curso. Em relação à população mundial, no ano de 1985, estudos indicavam haver 30 milhões de adultos com DM; em 2002, atingiu cerca de 173 milhões;

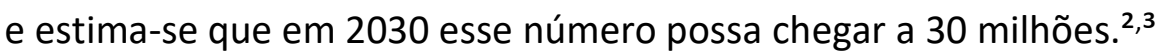

No Brasil, a prevalência de tal patologia na população, era de 11,7\% em 2012, sendo que as maiores prevalências foram obtidas nas regiões Sul $(12,5 \%)$ e Sudeste $(12,9 \%)$, sendo registrados, nos últimos anos, cerca de 124.795 indivíduos diabéticos no país. ${ }^{3}$

Ressalta-se que os frequentes episódios de hiperglicemia, algo comum nos pacientes diabéticos, podem lesionar órgãos e acarretar complicações severas, sendo importante a manutenção do adequado controle glicêmico, destacando-se o monitoramento da hemoglobina glicada A1C. ${ }^{2}$ Ademais, faz-se importante também controlar as demais comorbidades, uma vez que pacientes diabéticos apresentam maior risco de apresentar ataque cardíaco, acidente vascular encefálico, dentre outros problemas. $^{4}$

Apesar do controle glicêmico não ser uma ação de fácil alcance, estudos sugerem que a hiperglicemia está fortemente relacionada com as complicações do DM, o que compromete ainda mais o estado de saúde dos pacientes. ${ }^{5}$

Atualmente existe um arsenal terapêutico para o tratamento do DM, sendo que a insulina ainda é considerada a melhor alternativa terapêutica para controle glicêmico efetivo, quando seu uso for indicado, ${ }^{6}$ sendo também necessária a manutenção do autocuidado. $^{7}$

Portanto, para que os pacientes realizem as medidas de autocuidado e se tornem corresponsáveis pelo próprio tratamento é necessário que os profissionais de saúde realizem orientações sobre o DM, informando sobre diagnóstico, tratamento e controle da doença. Ao iniciar o tratamento com insulina o paciente também deve ser orientado sobre armazenamento, administração, descarte do medicamento e mensuração da glicemia capilar. Estudos indicam que quando os pacientes assumem o autocuidado e 0 realizam de forma adequada, previnem complicações da doença, aumentam a estimativa de vida e reduzem os custos com hospitalização. ${ }^{7}$ 
Com o intuito de contribuir para o uso correto da insulina, um programa de residência multiprofissional em saúde do idoso propôs a implantação do Serviço de Orientação Sobre o Uso de Insulina (SOSUI), com enfoque em idosos hospitalizados e que receberam recomendações de uso de insulina na alta hospitalar. O serviço também consistia na realização de interação mediada por telefone após a alta hospitalar, a fim de identificar dúvidas ou dificuldades dos pacientes em relação ao uso de insulina.

O presente estudo tem o objetivo de descrever as ações realizadas no SOSUI, bem como os resultados alcançados.

\section{METODOLOGIA}

Trata-se de um estudo exploratório com o intuito de levantar as possíveis contribuições do SOSUI, realizado no período de agosto de 2014 a fevereiro de 2015.

O estudo foi realizado em um hospital público geral, de referência para a rede de atenção à urgência e emergência do sistema de saúde em Minas Gerais. Atualmente esse hospital é composto por um total de 360 leitos. O hospital abrange o Programa de Residência Multiprofissional em Saúde do Idoso (PRMSI), que foi iniciado em março de 2010. O intuito é propiciar o aprendizado em serviço com enfoque na multiprofissionalidade e na integralidade dos pacientes.

\section{Descrição do serviço de orientação na alta}

Fase pré-alta hospitalar - o SOSUI foi implementado no setor de cirurgia vascular, onde os pacientes acompanhados pela equipe multiprofissional eram idosos ou adultos considerados frágeis, e com indicação de amputação devido a complicações do pé diabético.

Por fragilidade, entende-se como uma síndrome multifatorial que envolve fatores psicológicos, sociais, biológicos e cognitivos, os quais podem ocasionar maiores complicações, como hospitalizações, quedas, incapacidades e óbito. ${ }^{8}$

Cerca de uma semana antes da alta hospitalar, profissionais farmacêuticos realizaram a abordagem para identificação do grau de entendimento do paciente sobre a doença, e a experiência medicamentosa desses pacientes. Para isso utilizou-se um 
diálogo inicial no qual o paciente era convidado a relatar seu entendimento sobre o DM, a importância da insulina, o modo de uso, as possíveis crenças e dificuldades vivenciadas no uso do medicamento, a experiência prévia em relação ao armazenamento e à administração desse medicamento, e demais informações, como necessidade de ajuda para administrar o medicamento, analfabetismo, etilismo, polifarmácia, tabagismo e independência para as atividades da vida diária no período anterior à internação. Para isso foi utilizado um protocolo institucional não validado, elaborado a partir das recomendações de abordagem ao paciente segundo STRAND, ${ }^{9}$ bem como recomendações sobre abordagens educacionais aos pacientes diabéticos advindas de protocolos, tratados e guidelines nacionais e internacionais. ${ }^{2,10-12}$

Desse modo, na abordagem inicial, também identificaram-se os hábitos dos pacientes em relação ao manejo e à administração da insulina no período prévio à internação, sendo eles: armazenamento incorreto, considerando-se o armazenamento da insulina na porta da geladeira e em locais próximos ao congelador; homogeneização, considerando-se incorreto o número de homogeneização inferior a 20 vezes e agitação rápida; e administração incorreta, considerando-se o local de aplicação, tendo como inadequados os tecidos musculares. O estudo também avaliou o tipo de descarte da insulina e correlatos, considerando-se como inadequado o descarte no lixo comum.

Para identificação dos itens homogeneização e administração, foram fornecidos aos pacientes frascos de insulina, agulhas e seringas, a fim de que pudessem demonstrar essas técnicas aos aplicadores do questionário. Realizava-se uma abordagem prática individualizada, em que cada paciente era convidado a simular a administração de insulina em uma esponja, seguida de orientação educacional por meio de discussão e demonstração das técnicas de armazenamento e administração adequadas.

Essas informações eram registradas no prontuário eletrônico da instituição, sendo também registradas mensuração de glicemia em jejum e hemoglobina glicada realizadas no momento da admissão hospitalar ou até 48 horas de internação.

Durante a orientação, também foi entregue uma cartilha sobre a temática e um dispositivo de auxílio para aplicação da insulina. Em caso de identificação de baixa acuidade visual, foi elaborada estratégia para facilitar a administração da insulina, como etiquetas identificadoras para diferenciação entre as insulinas regular e NPH.

Nos casos em que os pacientes apresentavam comprometimento cognitivo que 
impossibilitasse 0 processo de comunicação/aprendizagem, o acompanhante responsável pela administração da insulina foi abordado.

Fase pós-alta hospitalar - após um período mínimo de quatro meses após a alta, realizou-se uma interação mediada por telefone para identificar possíveis mudanças nos relatos de armazenamento e administração da insulina pelos pacientes orientados no serviço. Também identificou-se, por meio da entrevista telefônica, relatos de realização de monitoramento glicêmico por meio da glicemia capilar em jejum, hemoglobina glicada e os respectivos valores, que foram informados pelos pacientes ou cuidador, sendo as informações coletadas registradas em planilha informatizada.

A escolha do período mínimo de quatro meses ocorreu ao considerar o tempo que seria necessário para que o paciente conseguisse consulta com endocrinologista na atenção primária, associada à disponibilidade dos farmacêuticos residentes para realização do contato telefônico.

\section{Critérios de inclusão e exclusão}

Foram incluídos no estudo todos os pacientes que receberam orientação sobre o uso de insulina pelo SOSUI, que receberam prescrição de alta com indicação de uso desse medicamento e que foram contatados na interação mediada por telefone. Desse modo, pacientes e/ou acompanhantes não contatados na interação mediada por telefone e aqueles que não forneceram informações sobre as mensurações de glicemia em jejum ou hemoglobina glicada após a alta hospitalar foram excluídos do estudo.

No momento da interação mediada por telefone, os pacientes foram informados sobre o interesse dos dados serem inseridos em um projeto de pesquisa, também sendo excluídos do estudo aqueles que não consentiram em participar.

\section{Coleta e análise dos dados}

Para coleta de dados, criou-se relatório informatizado com todos os dados registrados em prontuário eletrônico na fase pré-hospitalar, além de análise dos dados registrados na planilha informatizada sobre a interação mediada por telefone. Os dados foram registrados em planilha do software Microsoft Excel, sendo excluídos os pacientes 
que não atendiam aos critérios de inclusão, seguidos de realização de análise estatística univariada.

\section{Aspectos éticos}

A presente proposta de trabalho foi submetida ao Comitê de Ensino e Pesquisa da instituição, sendo aprovado pelo parecer $\mathrm{n}$ ㅇ 1.057.180.

\section{RESULTADOS}

No momento da alta foram orientados 51 pacientes e um cuidador, sendo que desse total somente 17 pacientes atenderam aos critérios de inclusão, sendo os demais, excluídos. Os principais critérios de exclusão adotados foram:

- Oito pacientes por número de telefone inexistente $(23,53 \%)$, informado pela companhia telefônica.

- Onze (32,35\%) não atenderam às ligações.

- Três $(8,82 \%)$ por ausência de contato telefônico registrado.

- Dois $(5,88 \%)$ por alta sem insulina.

- Dois $(5,88 \%)$ por não consentirem participar da pesquisa.

- $\operatorname{Três}(8,82 \%)$ devido à ocorrência de óbito.

- Cinco $(14,72 \%)$ por não relatarem valores de glicemia em jejum ou hemoglobina glicada realizados no período posterior à alta hospitalar.

Dos participantes, $76,47 \%$ (13) eram do sexo masculino e $23,53 \%$ (4) do sexo feminino. A idade variou de 36 a 98 anos, sendo que a média foi de aproximadamente 63 anos em ambos os sexos. Ressalta-se que um percentual de 58,82\% dos pacientes tinha idade acima de 60 anos. Em relação ao tempo de internação, este variou de 1 a 77 dias, obtendo-se uma média de 21 dias. O perfil dos participantes do estudo está disponível na Quadro 1.

O estudo avaliou também os aspectos sociais dos pacientes, sendo que 5,88\% (1) afirmaram ser analfabetos; $29,41 \%$ (5) etilista; $17,65 \%$ (3) tabagista; $52,94 \%$ (9) ter baixa adesão ao tratamento; 58,82\% (10) usuários de polifarmácia; 35,29\% (6) necessitavam 
de auxílio para administração dos medicamentos e 70,59\% (12) relatavam ser independentes para as atividades de vida diária.

Quadro 1 - Perfil demográfico dos pacientes que participaram do estudo

\begin{tabular}{|c|c|c|c|c|c|c|c|}
\hline $\begin{array}{c}\text { Código } \\
\text { do } \\
\text { pacien } \\
\text { te }\end{array}$ & $\begin{array}{c}\text { Polifarmá } \\
\text { cia }\end{array}$ & $\begin{array}{c}\text { Analfabetis } \\
\text { mo }\end{array}$ & $\begin{array}{c}\text { Etilism } \\
0\end{array}$ & $\begin{array}{c}\text { Tabagis } \\
\text { mo }\end{array}$ & $\begin{array}{c}\text { Auxílio na } \\
\text { administraç } \\
\text { ão dos } \\
\text { medicamen } \\
\text { tos }\end{array}$ & $\begin{array}{c}\text { Independên } \\
\text { cia para } \\
\text { AVDs }\end{array}$ & $\begin{array}{c}\text { Problema } \\
\text { s de } \\
\text { adesão } \\
\text { ao } \\
\text { tratamen } \\
\text { to }\end{array}$ \\
\hline 1 & 1 & 2 & 2 & 1 & 1 & 2 & 1 \\
\hline 2 & 1 & 2 & 1 & 2 & 1 & 2 & 2 \\
\hline 3 & 1 & 2 & 2 & 2 & 1 & 2 & 2 \\
\hline 4 & 1 & 2 & 2 & 2 & 1 & 2 & 1 \\
\hline 5 & 2 & $\mathrm{NI}$ & $\mathrm{NI}$ & $\mathrm{NI}$ & 2 & 1 & $\mathrm{NI}$ \\
\hline 6 & 1 & 2 & 2 & 2 & 2 & 1 & 1 \\
\hline 7 & 2 & 2 & 2 & 2 & 2 & 1 & 1 \\
\hline 8 & 1 & 2 & 2 & 2 & 1 & 2 & 2 \\
\hline 9 & 2 & 2 & 1 & 1 & 2 & 1 & 1 \\
\hline 10 & 2 & 2 & 2 & 2 & 2 & 1 & $\mathrm{NI}$ \\
\hline 11 & 2 & $\mathrm{NI}$ & 2 & 2 & 2 & 1 & $\mathrm{NI}$ \\
\hline 12 & 2 & $\mathrm{NI}$ & 1 & 1 & 2 & 1 & 1 \\
\hline 13 & 1 & $\mathrm{NI}$ & 2 & 2 & 2 & 1 & 2 \\
\hline 14 & 1 & 2 & 1 & 2 & 2 & 1 & 1 \\
\hline 15 & 1 & 1 & 2 & 2 & 1 & 1 & 1 \\
\hline 16 & 1 & $\mathrm{NI}$ & 1 & 2 & 2 & 1 & $\mathrm{NI}$ \\
\hline 17 & 2 & 2 & 2 & 2 & 2 & 1 & 1 \\
\hline
\end{tabular}

Legenda: 1 = Sim; 2 = Não; $\mathrm{NI}$ = não informado.

AVDs: Atividades da Vida Diária

Fonte: Elaborada pelas autoras - 2016

O estudo também comparou as informações fornecidas pelos pacientes sobre armazenamento, homogeneização, locais de aplicação e descarte da insulina antes de serem orientados e no contato pós-alta, que se encontram especificadas na Quadro 2. 
Quadro 2 - Comparação do conhecimento prévio do paciente sobre o manejo da insulina e o conhecimento após orientação farmacêutica

\begin{tabular}{|c|c|c|c|c|}
\hline \multicolumn{5}{|c|}{ ARMAZENAMENTO } \\
\hline & \multicolumn{2}{|c|}{ Internação } & \multicolumn{2}{|c|}{ Contato pós-alta } \\
\hline & $\mathrm{N}$ & $\%$ & $\mathrm{~N}$ & $\%$ \\
\hline Correto & 9,00 & 52,94 & 9,00 & 52,94 \\
\hline Incorreto & 4,00 & 23,53 & 8,00 & 47,06 \\
\hline Não informaram & 4,00 & 23,53 & 0,00 & 0,00 \\
\hline \multicolumn{5}{|c|}{ Especificação sobre o local de armazenamento incorreto } \\
\hline Porta da geladeira & 3,00 & 75,00 & 5,00 & 62,50 \\
\hline $\begin{array}{c}\text { Caixa de isopor na } \\
\text { geladeira }\end{array}$ & 1,00 & 25,00 & 0,00 & 0,00 \\
\hline Próximo ao congelador & & & 3,00 & 37,50 \\
\hline \multicolumn{5}{|c|}{ HOMOGENEIZAÇÃO } \\
\hline & \multicolumn{2}{|c|}{ Internação } & \multicolumn{2}{|c|}{ Contato pós-alta } \\
\hline & $\mathrm{N}$ & $\%$ & $\mathrm{~N}$ & $\%$ \\
\hline Correto & 6,00 & 35,29 & 12,00 & 70,59 \\
\hline Incorreto & 9,00 & 52,94 & 5,00 & 29,41 \\
\hline Não informaram & 2,00 & 11,76 & 0,00 & 0,00 \\
\hline \multicolumn{5}{|c|}{ Especificação das homogeneizações incorretas } \\
\hline Menos de 20 vezes & 5,00 & 55,56 & 5,00 & 100,00 \\
\hline Rapidamente & 3,00 & 33,33 & 0,00 & 0,00 \\
\hline Não homogeneizava & 1,00 & 11,11 & 0,00 & 0,00 \\
\hline \multicolumn{5}{|c|}{ LOCAL DE APLICAÇÃO } \\
\hline & \multicolumn{2}{|c|}{ Internação } & \multicolumn{2}{|c|}{ Contato pós-alta } \\
\hline & $\mathrm{N}$ & $\%$ & $\mathrm{~N}$ & $\%$ \\
\hline Correto & 8,00 & 47,06 & 16,00 & 94,12 \\
\hline Incorreto & 5,00 & 29,41 & 1,00 & 5,88 \\
\hline Não informaram & 4,00 & 23,53 & 0,00 & 0,00 \\
\hline \multicolumn{5}{|c|}{ Especificação do local de aplicação incorreto } \\
\hline Quadríceps & 1,00 & 20,00 & 0,00 & 0,00 \\
\hline Deltoide & 1,00 & 20,00 & 0,00 & 0,00 \\
\hline Parte interna da coxa & 3,00 & 60,00 & 0,00 & 0,00 \\
\hline Panturrilha & 0,00 & 0,00 & 1,00 & 100,00 \\
\hline
\end{tabular}


(conclusão)

\begin{tabular}{|c|c|c|c|c|c|}
\hline \multicolumn{7}{|c|}{ DESCARTE } \\
\hline & Internação & \multicolumn{3}{c|}{ Contato pós-alta } \\
\hline & $\mathrm{N}$ & $\%$ & $\mathrm{~N}$ & 88,24 \\
\hline Correto & 6,00 & 35,29 & 15,00 & 11,76 \\
\hline Incorreto & 7,00 & 41,18 & 2,00 & 0,00 \\
\hline Não informaram & 4,00 & 23,53 & 0,00 & 0,00 \\
\hline \multicolumn{7}{|c|}{ Local de descarte incorreto } \\
\hline Lixo comum & 7,00 & 100 & 100 & \\
\hline Considerando o total de pacientes que foram estabelecidos no contato pós-alta, sendo 100\% \\
\hline \multicolumn{7}{c|}{$=17$ pacientes. } \\
\hline
\end{tabular}

Fonte: Elaborada pelas autoras - 2016

Em relação ao perfil glicêmico dos participantes (Quadro 3), observa-se que houve melhora nos níveis de glicemia de jejum e hemoglobina glicada dos pacientes orientados pelo serviço.

Quadro 3 - Perfil glicêmico dos participantes do estudo no momento da internação e no contato pós-alta hospitalar

\begin{tabular}{|c|c|c|c|c|}
\hline $\begin{array}{c}\text { Código do } \\
\text { paciente }\end{array}$ & $\begin{array}{c}\text { Glicemia de } \\
\text { jejum } \\
\text { (internação) }\end{array}$ & $\begin{array}{c}\text { Glicemia de } \\
\text { jejum (contato } \\
\text { pós-alta) }\end{array}$ & $\begin{array}{c}\text { Hemoglobina glicada } \\
\text { (A1C) no início da } \\
\text { internação }\end{array}$ & $\begin{array}{c}\text { Hemoglobina } \\
\text { glicada pós-alta } \\
\text { hospitalar }\end{array}$ \\
\hline 1 & 84 & 360 & - & - \\
\hline 2 & 139 & 110 & 8,4 & 7,6 \\
\hline 3 & - & 57 & 11,9 & 8,30 \\
\hline 4 & 229 & 145 & 12,6 & 7 \\
\hline 5 & 199 & 89 & 7,4 & - \\
\hline 6 & 123 & 160 & 8,8 & - \\
\hline 7 & 367 & 90 & 11,1 & - \\
\hline 8 & 123 & 81 & 7,2 & 10 \\
\hline 9 & 256 & 145 & & -6 \\
\hline
\end{tabular}


(conclusão)

\begin{tabular}{|c|c|c|c|c|}
\hline $\begin{array}{c}\text { Código do } \\
\text { paciente }\end{array}$ & $\begin{array}{c}\text { Glicemia de } \\
\text { jejum } \\
\text { (internação) }\end{array}$ & $\begin{array}{c}\text { Glicemia de } \\
\text { jejum (contato } \\
\text { pós-alta) }\end{array}$ & $\begin{array}{c}\text { Hemoglobina glicada } \\
\text { (A1C) no início da } \\
\text { internação }\end{array}$ & $\begin{array}{c}\text { Hemoglobina } \\
\text { glicada pós-alta } \\
\text { hospitalar }\end{array}$ \\
\hline 10 & 231 & 120 & - & - \\
\hline 11 & 149 & 183 & 11,8 & - \\
\hline 12 & 171 & 170 & - & 7,8 \\
\hline 13 & 268 & 150 & 11 & 7,9 \\
\hline 14 & 132 & 86 & 10,8 & 7,6 \\
\hline 15 & 107 & 197 & 5,6 & 6,40 \\
\hline 16 & 367 & 90 & 11,1 & 7 \\
\hline 17 & 241 & 97 & 9,1 & 9,97 \\
\hline MÉDIA & 187,41 & 129,44 & & \\
\hline
\end{tabular}

Legenda: - = Ausência de resultado.

Fonte: Elaborada pelas autoras - 2016

\section{DISCUSSÃO}

No estudo foi evidenciada maior proporção de indivíduos do sexo masculino $(76,47 \%)$ orientados pelo serviço, fato também observado no estudo conduzido em seis capitais brasileiras que demonstrou uma prevalência de $42,6 \%$ de diabetes no sexo masculino. ${ }^{13}$ Entretanto, como a amostra estudada é reduzida, não se pode afirmar que haja maior prevalência do DM no sexo masculino na instituição em estudo.

De acordo com o perfil dos pacientes selecionados, verifica-se que a idade média e a proporção de pacientes com idade superior a 60 anos podem estar relacionadas com o fato de que o SOSUI foi desenvolvido dentro de um programa multiprofissional de atendimento a idosos, no qual essa faixa etária já é esperada. Além disso, ressalta-se que o intervalo de idade entre 55 a 74 anos é aquele em que os diabéticos têm maior risco de amputação (4,6 vezes) quando comparados à faixa etária de 34 a 55 anos, conforme evidenciou estudo realizado no Rio de Janeiro. O DM tem sua incidência aumentada com o envelhecimento populacional, as mudanças no estilo de vida, entre outros fatores citados anteriormente. ${ }^{14,15}$ 
Sabe-se que o grau de escolaridade, etilismo e tabagismo apresentam grande impacto na adesão ao tratamento, bem como na evolução clínica da doença.

Os dados sobre etilismo, tabagismo e analfabetismo foram registrados em prontuário informatizado conforme relato do próprio paciente, sendo que podem não ter sido informados com total fidelidade, ao considerar possível sentimento de inibição dos pacientes em fornecer tais dados. Entende-se que a identificação desses dados como de importância para o tratamento, pois são fatores que podem influenciar na adesão ao tratamento e na execução de funções de autocuidado relacionadas ao DM. Recomenda-se que além da identificação dos dados também sejam definidas as demais especificidades, como ano de escolaridade, número de maços de cigarro fumados por dia e quantidade de álcool ingerido por semana, pois isso permite diferenciar os pacientes em subgrupos, conforme hábitos de vida e desenvolvimento de estratégias educacionais específicas.

A adesão ao tratamento está relacionada a fatores como grau de instrução, nível socioeconômico, polifarmácia, desconhecimento sobre a doença e suas complicações, bem como dificuldade de acesso aos serviços de saúde. Sabe-se que pacientes com baixa adesão têm maiores complicações devido ao DM. Já aqueles com boa adesão ao tratamento apresentam melhor controle glicêmico e consequente controle nas complicações do DM. O reflexo da baixa adesão ao tratamento está relacionado a amputações, hospitalizações e elevação nos gastos com saúde. ${ }^{16,17}$ Baixa adesão e polifarmácia também foram predominantes entre os pacientes entrevistados, e são aspectos que devem ser melhor investigados.

A administração de medicamentos é uma atividade instrumental muito complexa, principalmente quando envolve a insulina. Como pacientes com DM muitas vezes apresentam baixa acuidade visual, problemas cognitivos, déficit na compreensão do processo saúde-doença e são usuários de polifarmácia, tal atividade se torna um fator limitante para a adesão ao tratamento, portanto sendo necessária a ajuda de cuidadores para administração dos medicamentos. Dos participantes do estudo, $70,59 \%$ afirmaram ser independentes para as atividades de vida diárias básicas e instrumentais, sendo que $35,29 \%$ referiram necessitar de auxílio para administração dos medicamentos.

Entende-se que a independência do idoso para determinadas atividades o 
caracteriza como independente para atividades instrumentais, com exceção do gerenciamento da farmacoterapia, atividade que geralmente é muito complexa por exigir boa acuidade visual para o entendimento das caixas de medicamentos e cores, capacidade de leitura e boa cognição.

Conforme recomendação da Sociedade Brasileira de Diabetes (SBD), para que seja alcançado o controle glicêmico é importante que os pacientes com DM passem por processos de orientação sobre preparo e aplicação da insulina. ${ }^{18}$

O processo de educação em saúde deve abranger também explicações sobre armazenamento e homogeneização da insulina. ${ }^{11} \mathrm{Em}$ relação às técnicas de armazenamento e administração da insulina, identificou-se que 47,06\% pacientes relatavam armazená-la incorretamente durante a interação mediada pelo telefone, sendo que desses pacientes $62,50 \%$ relatavam armazenar próximo à porta da geladeira, e 37,50\% próximo ao congelador.

$\mathrm{O}$ armazenamento da insulina em temperaturas inferiores a $2{ }^{\circ} \mathrm{C}$ está associado com o risco de congelamento, assim como o armazenamento na porta da geladeira, devido às oscilações de temperatura e mobilidade do frasco, podendo resultar em perda da estabilidade do medicamento. ${ }^{2}$

Entretanto, ao considerar que a SBD recomenda que a insulina lacrada deva ser armazenada na geladeira à temperatura de $2-8{ }^{\circ} \mathrm{C}$ até o vencimento, sendo que as insulinas abertas podem ser armazenadas refrigeradas ou à temperatura ambiente (máximo $30^{\circ} \mathrm{C}$ ) por um período de quatro semanas, identifica-se que o armazenamento na geladeira ou fora dela, desde que não exposta ao calor excessivo ou ao congelamento, não apresenta riscos de comprometimento da efetividade da farmacoterapia quando utilizada em curto prazo. $^{2}$

Infere-se que mesmo orientados, ao identificarem o não comprometimento da farmacoterapia, os pacientes podem ter optado pelo armazenamento em locais considerados mais práticos, desde que o medicamento não seja congelado. Outra possibilidade é o fato de a intervenção educacional sobre o armazenamento não ter sido efetiva, considerando que o paciente tenha centrado maior atenção em outras informações de interesse, como as técnicas de administração. Também se destaca que as informações sobre técnicas de administração e homogeneização podem ter chamado maior atenção por contemplar abordagens práticas, o que não foi possível realizar ao se 
falar sobre armazenamento.

Entretanto, o presente estudo não investigou o tempo médio de armazenagem da insulina em locais incorretos ou a existência ou não de frascos considerados como estoques nesses locais, o que poderia comprometer os resultados clínicos positivos do tratamento, sendo essa uma das limitações do estudo.

Em relação à homogeneização, ela deve ser realizada com movimentos suaves de 10 a 20 vezes para evitar surgimento de bolhas que possam dificultar a aspiração da dose corretamente e a administração. ${ }^{2}$ Quando questionados sobre o processo de homogeneização da insulina, foi observado que 70,59\% dos participantes do estudo realizavam o procedimento corretamente após a alta hospitalar, comparado com 35,29 \% no período da internação, o que sugere mudança de hábitos dos pacientes em relação a esse procedimento.

Em relação aos locais de aplicação, foi verificada melhora na proporção dos participantes que relatam realizar administração correta após as medidas de educação em saúde. Somente um paciente dentre os orientados relatou administrar a insulina de maneira incorreta, sendo novamente orientado quanto aos locais de aplicação. Entretanto, nas informações obtidas por meio do contato telefônico existe a limitação do entrevistador não solicitar o paciente que simule a técnica, sendo essa informação obtida somente pelo relato. Por outro lado, ao identificar o relato correto, entende-se a ocorrência de uma conscientização dos pacientes sobre a técnica adequada de administração do medicamento após a alta hospitalar.

Em relação às informações de descarte da insulina, também foi observada melhora nos hábitos de descarte após a alta hospitalar, o que reflete a melhora do impacto ambiental e a segurança nesse processo.

Foi evidenciado que, dentre os pacientes apresentavam uma hemoglobina glicada média de 9,97\%, sendo esse valor médio reduzido para 7,0\% após a alta hospitalar. Isso indica melhora do controle glicêmico pelos pacientes, o que pode ser influenciado pelas experiências de adoecimento, internação e amputação decorrente de complicações do diabetes, associadas com o fato de os pacientes terem também recebido orientações educacionais sobre o uso da insulina, favorecendo melhor entendimento sobre o uso desse medicamento.

Ressalta-se que alguns pacientes não tiveram o exame de hemoglobina glicada 
realizado na internação e/ou pós-alta hospitalar, ou não souberam informar. Isso reforça a necessidade do fortalecimento das estratégias de monitoramento e fortalecimento do autocuidado no âmbito da atenção primária.

Conforme evidenciado no estudo de revisão da literatura, a melhor ferramenta para avaliar a adesão ao tratamento em pacientes diabéticos é através do controle glicêmico. Assim como em outros estudos, nos quais foram comparados os valores de glicemia e A1C antes e após as medidas de educação em saúde, observou-se melhora nos parâmetros glicêmicos, sendo que tal melhora pode indicar melhor adesão ao tratamento. ${ }^{7}$

Segundo o estudo conduzido na Jordânia, que comparou grupos de pacientes acompanhados por farmacêuticos clínicos e grupo sem acompanhamento farmacoterapêutico, foi evidenciado que os pacientes submetidos à assistência farmacêutica apresentaram melhores: controle glicêmico; mudança nos hábitos de vida; e conhecimento sobre DM, medicamentos e adesão ao tratamento, indicando a importância do farmacêutico clínico na assistência ao paciente diabético. ${ }^{19}$

Observa-se que as Unidades Básicas de Saúde (UBS) têm papel importante na promoção e na educação dos pacientes com DM através da realização de trabalhos voltados para o fornecimento de informações sobre a doença e suas complicações, o incentivo ao autocuidado, as mudanças no estilo de vida, bem como o treinamento da equipe de enfermagem, o gerenciamento da farmacoterapia por meio da assistência farmacêutica e o acompanhamento das glicemias. ${ }^{20}$

Contudo, entende-se que o processo de educação sobre o DM deve ser iniciado imediatamente após o diagnóstico da doença, uma vez que a adoção de medidas adequadas previne complicações irreversíveis. Nos casos de pacientes internados em instituições hospitalares, vale ressaltar a importância de realizar a transição do cuidado de maneira efetiva, fazendo com que o paciente continue sendo assistido e acompanhado nas UBS.

O estudo teve como limitações o viés das informações que foram provenientes de relatos dos pacientes e de registros em prontuários eletrônicos, sendo que não puderam ser confirmadas presencialmente, assim como o considerável número de sujeitos excluídos.

Em relação ao SOSUI, verifica-se a necessidade de reformulação do serviço para 
que ocorra maior sucesso em relação ao número de pacientes contatados (identificação do contato telefônico durante a entrevista realizada na internação hospitalar, e não por meio da ficha de registro no prontuário). Contudo, foi possível evidenciar que houve melhora no perfil de utilização da insulina pelos pacientes atendidos nesse serviço.

\section{CONSIDERAÇÕES FINAIS}

A implementação do SOSUI serviu como importante ferramenta no processo de educação em saúde a respeito do diabetes mellitus (DM) para os pacientes acompanhados pelo Serviço de Farmácia Clínica, proporcionando melhora no gerenciamento da farmacoterapia com insulina, melhor conhecimento sobre o DM E suas complicações e a importância da adesão ao tratamento.

\section{REFERÊNCIAS}

1. World Health Organization (Estados Unidos). Definition, diagnosis and classification of Diabetes Mellitus and its complications. Geneva,1999.

2. Sociedade Brasileira de Diabetes (Brasil). Diretriz da Sociedade Brasileira de Diabetes 2013-2-14. Rio de Janeiro, SBD, 382p.

3. DATASUS [internet]. Brasília (DF): Ministério da Saúde, 2016 [citado 2016 mar 03]. Disponível em: http://datasus.gov.br.

4. Diabetes complications. Rochester: Health Letter Mayo Clinic, 2012. 3p.

5. Abott LW, Ira MH. Vascular complications and diabetes: current therapies and future challenges. J Ophthalmology, 2012. 14p.

6. Taylor JR, Campbel KM. Insulin analogs for the management of type 2 diabetes. Am J Health Syst Pharm. 2013 Feb 15;70(4):320-34.

7. Zareban I, Niknami S, Hidarnia A, Rakhshani F, Shamsi M, Karimi M. Effective intervention of self-care on glycaemia control in patients with type 2 diabetes. J. Iran Red Crescent Med, 2014;16(12):8311.

8. Storti LB, Farício-Webe SCC, Kusumota L, Rodrigues RAP, Marques S. Frailty of elderly patients admitted to the medical clinic of an emergency unit at a general tertiary hospital. Text Context Nursing, Florianópolis, 2013 Abr-Jun; 22(2): 452-9.

9. Strand LM, Cipolle RJC, Morley PC, Frakes MJ. The impact of pharmaceutical care practice on the practitioner and the patient in the ambulatory practice setting: twenty-five years of experience. Curr Pharm Des, 10: 3987-4001, 2004.

10. Frid A, Hirsch L, Hicks D, Kreugel G, Liersch J, Letondeur C et al. The third injection technique workshop In Athens (TITAN). Diabetes \& Metabolism 36 (2010) S19-S29. 
11. Frid A, Hirsch L, Gaspar R, Hicks D, Kreugel G, Liersch J et al. New injection recommendations for patients with diabetes. Diabetes \& Metabolism 36 (2010) S3-S18.

12. American Diabetes Association (ADA). Standards of medical care in diabetes -2014 . Diabetes Care. 2014;37(suppl 1):S14-S80.

13. Iser BPM, Stopa SR, Chueiri PS, Szwarcwald CL, Malta DC, Monteiro HCO et al. Prevalência de diabetes autorreferido no Brasil: resultados da Pesquisa Nacional de Saúde 2013. Epidemiol. Serv. Saúde, Brasília, 24(2): 305-314, abr-jun 2015.

14. Schmidt MI, Duncan BB, Hoffmann JF, Moura L, Malta DC, Carvalho RMSV. Prevalência de diabetes e hipertensão no Brasil baseada em inquérito de morbidade autorreferida, Brasil, 2006. Rev Saúde Pública 2009;43(Supl. 2):74-82.

15. Tavares DMS, Dias FA, Araújo RL, Pereira GA. Perfil de clientes submetidos a amputações relacionadas ao diabetes mellitus. Rev Bras Enferm, Brasília 2009 nov-dez; 62(6): 825-30.

16. Asche C, La Fleur J, Conner C. A review of diabetes treatment adherence and the association with clinical and economic outcomes. Clinical Therapeutics/Volume 33, Number 1, 2011.

17. Melo EM, Teles MS, Teles RS, Barbosa IV, Studart RMB, Oliveira MM. Avaliação dos fatores interferentes na adesão ao tratamento do cliente portador de pé diabético. Revista de Enfermagem Referência, III Série - n. 5 - Dez. 2011.

18. Dias VP, Silveira DT, Witt RR. Educação em saúde: o trabalho de grupo em Atenção Primária. Rev. APS, v. 12, n. 2, p. 221-227, abr./jun. 2009.

19. Ruba AW, Omar AAK, Abla MA. Impact of pharmaceutical care interventions on glycemic control and other health-related clinical outcomes in patients with type 2 diabetes: randomized controlled trial. Diabetes \& Metabolic Syndrome: Clinical Research \& Reviews 9 (2015) 271-276.

20. Silva RKL, Junior AM. Atenção à saúde no diabetes mellitus na perspectiva da estratégia de saúde da família: uma revisão integrativa. Revista Eletrônica Gestão e Saúde 1 (2015) 152-167.

Submissão: dezembro de 2016.

Aprovação: setembro de 2018. 